\title{
SPATIAL STATISTICAL ANALYSIS OF THE POROUS MEDIUM EXTRACTED FROM VIRTUAL PACKED GRAINS WITH RANDOM SIZES
}

\author{
*Yu Song ${ }^{1}$, Junichiro Takeuchi ${ }^{1}$, Yuto Takeuchi ${ }^{1}$ and Masayuki Fujihara ${ }^{1}$ \\ ${ }^{1}$ Graduate School of Agriculture, Kyoto University, Japan
}

*Corresponding Author, Received: 10 Nov. 2021, Revised: 19 Nov. 2021, Accepted: 27 Nov. 2021

\begin{abstract}
Hydraulic properties such as water retention properties and permeability, which are macro characteristics of porous media, are determined in a bottom-up manner by pore properties including pore shape, pore-size distribution, and pore connectivity. Hydraulic properties could be reproduced from local physical phenomena such as capillarity in the pores using a pore-network model. Spatial distribution of pores could affect these hydraulic properties. In this research, global and local Moran's indexes, which are spatial statistics, are used to analyze the spatial distribution of pores in porous media packed with both single-size and various-size grains, to find the global spatial autocorrelation in the pore distribution. The obtained results show that regardless of the grain-size distribution, almost all the global Moran's indexes are just below 0.5, which indicates there are some clusters with respect to the pore-size distribution. From the local Moran's indexes, it was found that the numbers of significant PBs in $\mathrm{HH}$ category at the 5\% statistically significant level are obviously higher than LH, LL and HL regions, which indicates that large PBs tend to distribute around large PBs. All of these imply that hydraulic properties of porous media could be affected by the spatial structure of pores.
\end{abstract}

Keywords: Water retention property, Pore-network, Spatial statistics, Global and local Moran's indexes

\section{INTRODUCTION}

It is well known that soil water relations are an important aspect of the soil environment of the plant. The hydraulic properties of soils such as water retention properties and permeability, which are macro characteristics of porous media, play an important central role in determining the movement and storage of water in soil and thus have a large influence on crop growth.

\section{RESEARCH SIGNIFICANCE}

Soil water retention and transport properties are altered in response to changes in pore properties including pore shape, pore-size distribution, and pore connectivity [1]. Pore-network models could be utilized to reproduce the hydraulic properties from local physical phenomena such as capillarity in the pores $[2,3]$. It was presented that the water retention property of the original pore-network model extracted from a porous medium model is different from that of pore-network models generated randomly based on the same pore-size distribution, which suggests that pores have a certain spatial structure in the porous medium [4].

In this research, we used the spatial statistics called global and local Moran's indexes [5] to analyze the spatial pore-size distribution of porous media packed with both single-size and various-size grains, to find the global spatial autocorrelation in the pore distribution.

\section{SPATIAL AUTOCORRELATION}

Spatial autocorrelation can be defined in the phenomenon that occurs when the spatial distribution of the variable of interest exhibits a systematic pattern. Moran's I statistics are one of the statistical and computational methods for examining spatial autocorrelation on a network, that is, the correlation between attribute values of the same kind at different locations on a network or on different subnetworks (including line segments and links) forming the network [6]. Moran's I statistics include two types: global Moran's I statistic and local Moran's I statistic. The former shows the average level of spatial autocorrelation over the whole network; the latter examines the correlation between the attribute value of a specific network point and those of its surrounding network points.

\subsection{Global spatial autocorrelation}

The global Moran's I statistic on a network, network global Moran's $I$ statistic, is used for global spatial autocorrelation, which is defined as:

$$
I=\frac{\sum_{i=1}^{n} \sum_{j=1}^{n} w_{i j}\left(x_{i}-\bar{x}\right)\left(x_{j}-\bar{x}\right)}{m_{2} S_{0}},
$$


where $n$ is the number of the data, $x_{i}$ is the attribute value that denotes the value taken on by the variable $x$ of interest at location $i$ and $\bar{x}$ denotes the mean of these values, which is defined as follows:

$$
\bar{x}=\frac{1}{n} \sum_{i=1}^{n} x_{i},
$$

$m_{2}$ and $S_{0}$ are defined as:

$$
\begin{aligned}
& m_{2}=\frac{1}{n} \sum_{i=1}^{n}\left(x_{i}-\bar{x}\right)^{2}, \\
& S_{0}=\sum_{i=1}^{n} \sum_{j=1}^{n} w_{i j} .
\end{aligned}
$$

In the present study, $x_{i}$ is the diameter of the pore body (PB) whose index is $i$, and $n$ is the number of all PBs in the interest pore-network.

Spatial autocorrelation statistics deal with the correlation between nearby attribute values. $w_{i j}$ denotes the elements of the spatial weights matrix $W$ corresponding to the location pair $(i, j)$ and can formalize the nearness mathematically. In this research, $w_{i j}=1$ if network points are adjacent; otherwise, $w_{i j}=0$.

When $I$ is positive, it suggests a positive autocorrelation; when $I$ is negative, it suggests a negative autocorrelation. Statistical test is based on $Z_{I}$ values, computed by subtracting $E[I]$ from $I$ and dividing it by the standard deviation of $I$, which is given by:

$$
Z_{I}=\frac{I-E[I]}{\sqrt{\operatorname{Var}(I)}} \sim N(0,1) .
$$

The expected value and variance of $I$ under the null hypothesis of the normal variate spatial randomness are derived as:

$$
\begin{aligned}
& E[I]=-\frac{1}{n-1}, \\
& \operatorname{Var}(I)=\frac{n^{2} S_{1}-n S_{2}+3 S_{0}^{2}}{\left(n^{2}-1\right) S_{0}^{2}}-\{E[I]\}^{2}, \\
& S_{1}=\frac{1}{2} \sum_{i=1}^{n} \sum_{j=1}^{n}\left(w_{i j}+w_{j i}\right)^{2}, S_{2}=\frac{1}{2} \sum_{i=1}^{n}\left(w_{i^{*}}+w_{*_{i}}\right)^{2}, \\
& w_{i^{*}}=\sum_{j=1}^{n} w_{i j}, w_{*_{i}}=\sum_{j=1}^{n} w_{j i} .
\end{aligned}
$$

\subsection{Local Spatial Autocorrelation}

The local Moran's I statistic can examine the correlation between the attribute value of a specific network point and those of its surrounding network points, which is defined as follows:

$$
I_{i}^{L}=\frac{\left(x_{i}-\bar{x}\right)}{m_{2}} \sum_{j=1}^{n} w_{i j}\left(x_{j}-\bar{x}\right) .
$$

This equation can express both the deviation from the average of the point's own value and that of the surrounding points'. That means, $I^{L}$ is positive when both of them are greater than or less than the average, and $I^{L}$ is negative if either one is greater or smaller than the average.

In terms of the network global Moran's I statistic, this equation is written as:

$$
I=\sum_{i=1}^{n} I_{i}^{L} / S_{0}
$$

This equation shows that the global Moran's I statistic is proportional to the average of network local Moran's I statistics over all network points.

The same as those of the global Moran's I statistic, the expected value and variance of $I^{L}$ under the null hypothesis of no global spatial autocorrelation, which can be utilized for identifying statistically significant points, are derived as:

$$
Z_{I_{i}^{L}}=\frac{I_{i}^{L}-E\left[I_{i}^{L}\right]}{\sqrt{\operatorname{Var}\left(I_{i}^{L}\right)}} \sim N(0,1),
$$

where

$$
\begin{aligned}
& E\left[I_{i}^{L}\right]=-\frac{w_{i^{*}}}{n-1}, \\
& \operatorname{Var}\left(I_{i}^{L}\right)=\frac{w_{i(2)}\left(n-b_{2}\right)}{n-1}+\frac{2 w_{i(k h)}\left(2 b_{2}-n\right)}{(n-1)(n-2)}-\{E[I]\}^{2},
\end{aligned}
$$

and

$$
\begin{aligned}
& w_{i(2)}=\sum_{j=1}^{n} w_{i j}^{2}, w_{i(k h)}=\frac{1}{2} \sum_{k=1}^{n} \sum_{h=1}^{n} w_{i k} w_{i h}, \\
& b_{2}=\frac{m_{4}}{m_{2}^{2}}, m_{4}=\frac{1}{n} \sum_{i=1}^{n}\left(x_{i}-\bar{x}\right)^{4} .
\end{aligned}
$$




\subsection{Moran Scatter Plot}

Spatial distribution of the attribute values can be visualized through classification by the scatter plot with the original attribute values on the horizontal axis and the surrounding values on the vertical axis [7].

In the Moran scatter plot, the attribute values are divided into four quadrants, each of which represents a different kind of spatial association: Spatial categories in the upper right $(\mathrm{HH}$, high-high) and lower left (LL, low-low) quadrants, and spatial categories in the lower right (HL, high-low) and upper left (LH, low-high) quadrants.

\section{NUMERICAL EXPERIMENTS}

\subsection{Pore-network Models}

Pore-network models extracted from porous media are utilized to conduct the numerical experiments in this research. The virtual porous media are prepared by using the discrete element method, which are composed of various kinds of spherical grains packed randomly in the cubic containers. The method used for pore network extraction is known as the watershed segmentation algorithm, which has been popular these days due to the effectiveness in working with porous media images of any porosity and its easier implementation $[8,9]$. In this research, we put SNOW algorithm [10], an efficient algorithm for extracting networks using only standard image analysis techniques, into implementation. Details on network extraction using this method can be found in [10].

A pore-network consists of pore bodies (PBs) and pore throats (PTs). PBs are relatively large, however, PTs that connect two PBs are relatively small. To make networks sufficiently large that exceed the representative element volume (REV), we tried packing spherical grains from 1000 to 7000 and extracted the pore networks under the conditions of the voxels from $200^{3}$ to $700^{3}$. By observing histograms of the frequency distributions of pore sizes and coordinate number, the number of PTs that a single PB connects, we find that when the number of grains is larger than 2000 and the voxels exceed $500^{3}$, packed porous media and the extracted networks are sufficiently large and resolved for numerical experiments.
In this research we packed porous media withfour grain size distributions (A-D) and three different samples were made in each grain size distribution: Sample A1-3 are packed with single-size spherical grains; the others are packed with uniformly distributed spherical grains. The numbers of grains all exceed 3000, and we choose voxels of $600^{3}$ to get more delicate models. The sample holders $(\mathrm{mm})$ of them are A- $44.1^{3}, \mathrm{~B}-32.6^{3}, \mathrm{C}-45.1^{3}$ and D-48. $1^{3}$.

Table 1 shows the properties of packed porous media and the extracted pore networks. Figure 1 shows the virtual porous media and pore-networks of A1 and D1. The former is packed with single-size spherical grains, and the latter is packed with uniformly distributed spherical grains. To eliminate the influence of boundary PBs and PTs, which tend to be irregalarly large, we make the frequency distributions of pore-sizes and coordinate number of the internal pores. Figure 2 shows those frequency distributions of D1-3 as an example, and it was confirmed that similar pore-size and coordinate number distributions were obtained from different grain-packing in the cubic container. Moreover, the following numerical experiments are all applied on the internal pore-network models.

Table 1 Properties of porous media and porenetworks

\begin{tabular}{|c|c|c|c|c|}
\hline $\begin{array}{c}\mathrm{N} \\
\mathrm{A} \\
\mathrm{M} \\
\mathrm{E}\end{array}$ & $\begin{array}{l}\text { Size of } \\
\text { grains } \\
(\mathrm{mm})\end{array}$ & $\begin{array}{l}\text { No. } \\
\text { of } \\
\text { grains }\end{array}$ & $\begin{array}{c}\text { No. } \\
\text { of } \\
\text { PB-internal/ } \\
\text { PB-all }\end{array}$ & $\begin{array}{c}\text { No. } \\
\text { of } \\
\text { PT-internal/ } \\
\text { PT-all }\end{array}$ \\
\hline A1 & 3 & 3615 & $9720 / 11441$ & $34255 / 35976$ \\
\hline A2 & 3 & 3603 & $9693 / 11450$ & $33893 / 35650$ \\
\hline A3 & 3 & 3619 & $9753 / 11551$ & $34256 / 36054$ \\
\hline B1 & $\begin{array}{l}1.77- \\
2.57\end{array}$ & 3728 & $9765 / 11530$ & $33956 / 35721$ \\
\hline B2 & $\begin{array}{l}1.77- \\
2.57\end{array}$ & 3725 & $9749 / 11484$ & $34111 / 35864$ \\
\hline B3 & $\begin{array}{l}1.77- \\
2.57\end{array}$ & 3714 & $9663 / 11422$ & $33727 / 35486$ \\
\hline $\mathrm{C} 1$ & $2-4$ & 3557 & $9280 / 11054$ & $32103 / 33727$ \\
\hline $\mathrm{C} 2$ & $2-4$ & 3525 & $9204 / 10907$ & $31592 / 33293$ \\
\hline $\mathrm{C} 3$ & $2-4$ & 3549 & $9336 / 11000$ & $32009 / 33823$ \\
\hline D1 & $1-5$ & 3342 & $7949 / 9417$ & $27468 / 28652$ \\
\hline D2 & $1-5$ & 3301 & $7787 / 9304$ & $26782 / 28299$ \\
\hline D3 & $1-5$ & 3311 & $7857 / 9505$ & $27092 / 29024$ \\
\hline
\end{tabular}




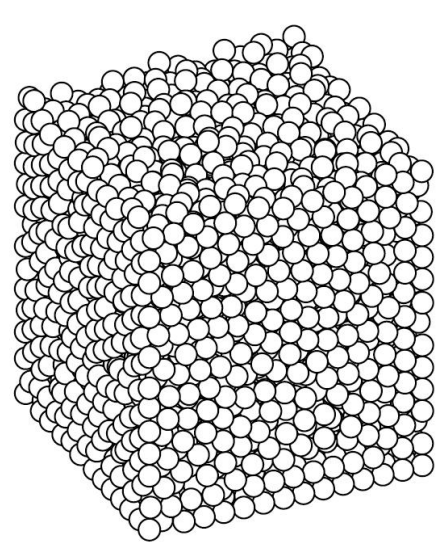

(a) Packed grains of A1

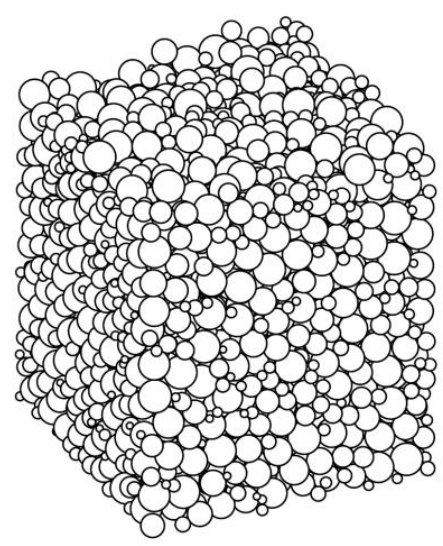

(c) Packed grains of D1

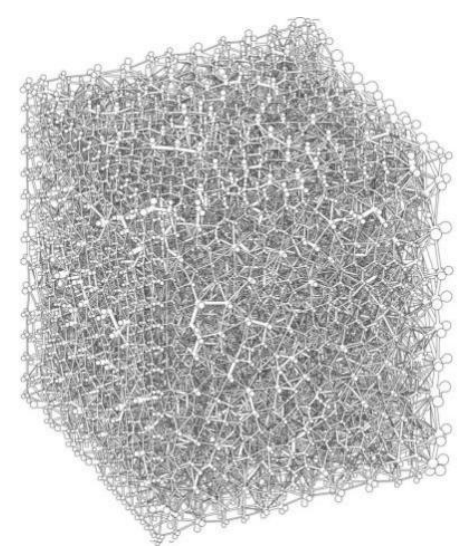

(b) Pore-network of A1

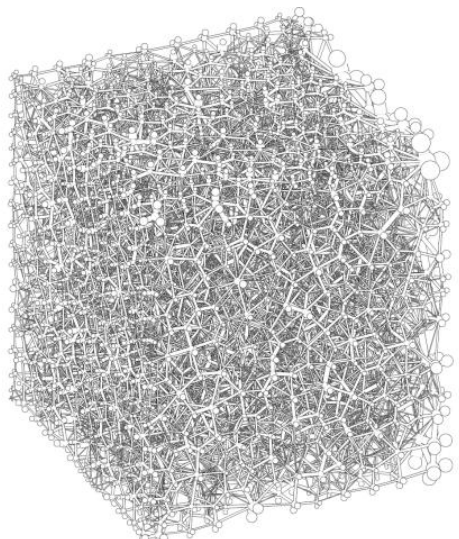

(d) Pore-network of D1

Fig. 1 Virtual porous media and pore-networks (A1, D1)

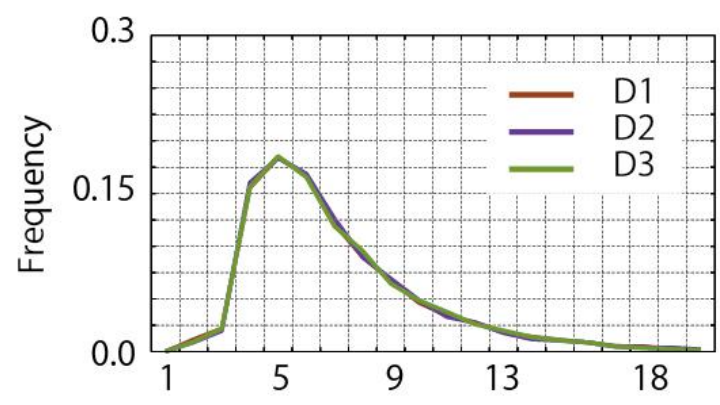

(a) Coordinate number (-)

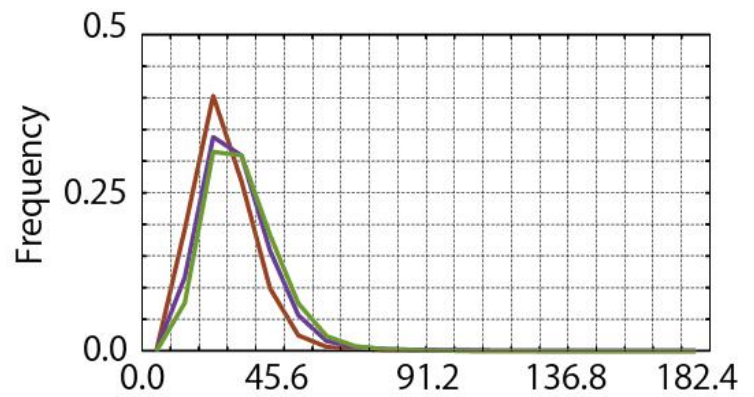

(c) Pore throat length $(0.1 \mathrm{~mm})$

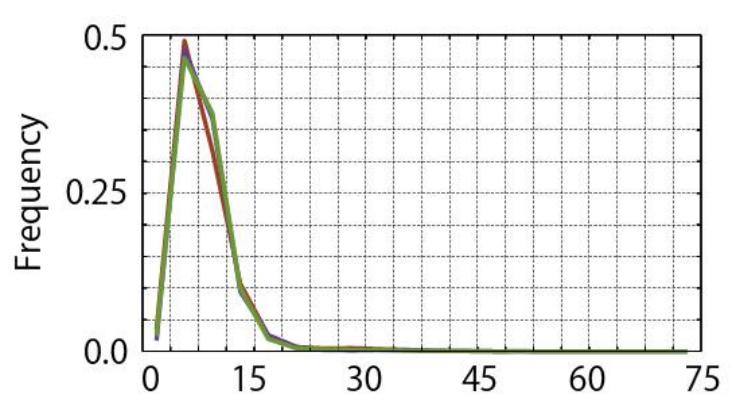

(b) Pore body radius $(0.1 \mathrm{~mm})$

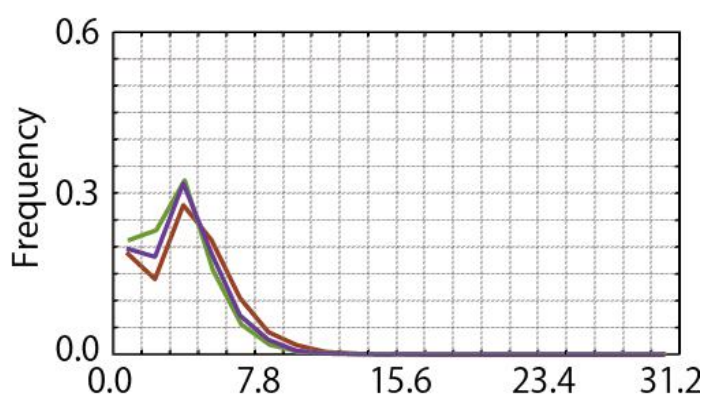

(d) Pore throat radius $(0.1 \mathrm{~mm})$

Fig. 2 Frequency of coordinate number and pore-sizes (D1-3, Internal) 


\subsection{Results}

The Global Moran's I of internal pore-network models are shown in Table 2 as well as the $Z$-score and $P$-value of them. When the results of $I$ distribute almost 0 , it indicates the normal variate spatial randomness and shows that pore-size distributions are random ones and that those has no global spatial autocorrelation. When there are some clusters with respect to the pore-size, the value increases upto 1 depending on the cluster size.

The Global Moran's I of A1-3, B1-3, C1-3, and D1-3 shows relatively large spatial autocorrelations, and the null hypothesis that there is no spatial autocorrelation was rejected at a significant level of $1 \%$, which indicates that there are some clusters of larger or smaller PBs in the porous media.

Regarding local Moran's $I$, the numbers of significant PBs at the 5\% statistically significant level in each category are shown in Table 2. Figure 3 denotes the Moran scatter plots of A1, B1, C1 and D1; Figure 4 shows the spatial distributions of categories for both all PBs and significant PBs of A1 and D1.

As we can see in Fig. 3, the numbers of statistically significant $\mathrm{PBs}$ in $\mathrm{HH}$ region are obviously higher than LH, LL and HL, and the numbers of significant PBs in LL and HL regions are extremely small, which indicates that large PBs tend to distribute around large PBs.

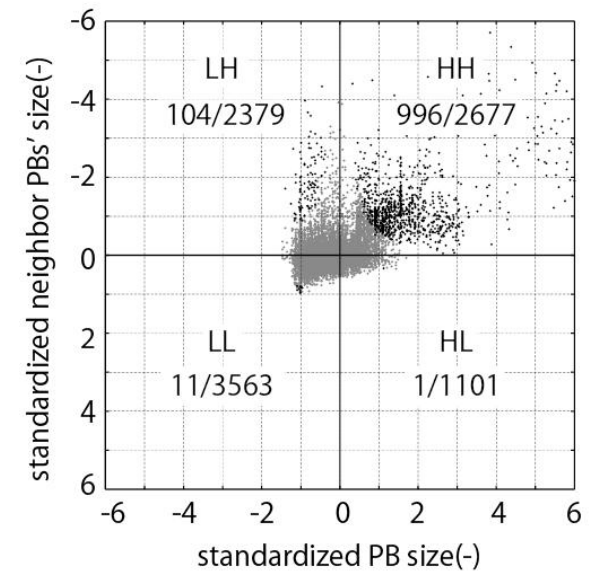

(a) A1

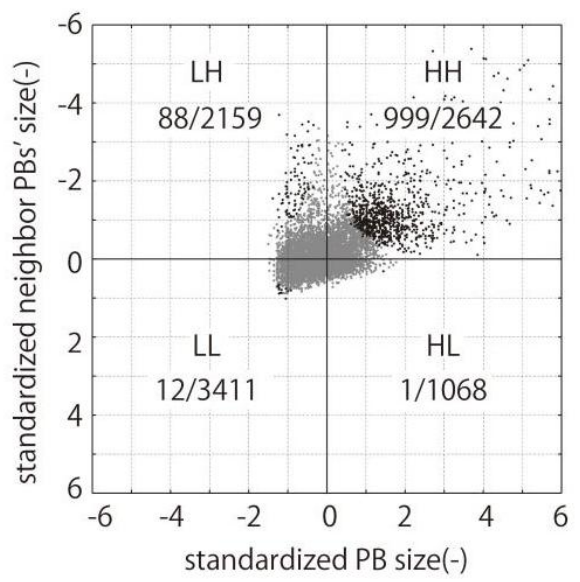

(c) $\mathrm{C} 1$

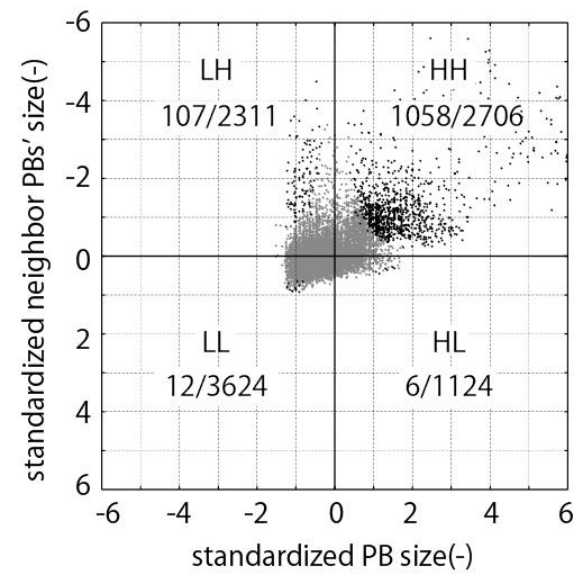

(b) B1

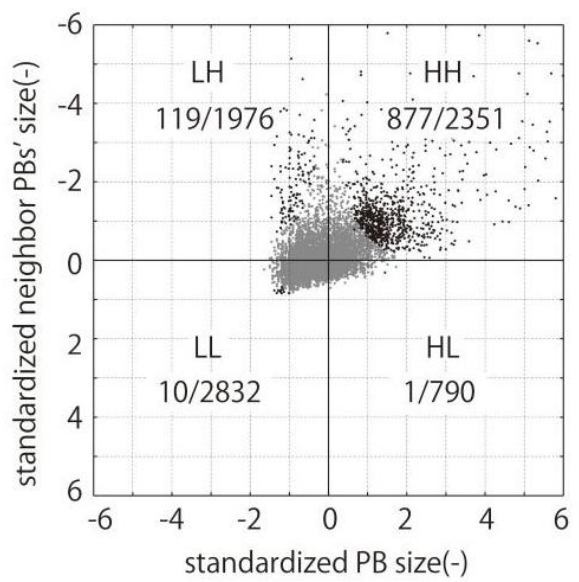

(d) D1

Fig. 3 Moran scatter plot 
Table 2 Global Moran's I and number of significant/all PBs-Internal in each category

\begin{tabular}{cccc|cccc}
\hline \multirow{2}{*}{ Pore-networks } & \multicolumn{3}{c|}{ Global Moran } & \multicolumn{4}{c}{ Local Moran } \\
& $I$ & $Z$-score & $P$-value & HH & LH & LL & HL \\
\hline A1 & 0.473 & 29.77 & Neary 0 & $996 / 2677$ & $104 / 2379$ & $11 / 3563$ & $1 / 1101$ \\
A2 & 0.488 & 30.57 & Neary 0 & $920 / 2700$ & $91 / 2346$ & $2 / 3566$ & $2 / 1081$ \\
A3 & 0.482 & 30.30 & Neary 0 & $942 / 2807$ & $89 / 2321$ & $4 / 3501$ & $0 / 1124$ \\
B1 & 0.498 & 31.57 & Neary 0 & $1058 / 2706$ & $107 / 2311$ & $12 / 3624$ & $6 / 1124$ \\
B2 & 0.489 & 30.89 & Neary 0 & $1030 / 2744$ & $105 / 2250$ & $7 / 3632$ & $2 / 1123$ \\
B3 & 0.495 & 31.18 & Neary 0 & $1022 / 2769$ & $91 / 2250$ & $31 / 3601$ & $2 / 1043$ \\
C1 & 0.474 & 29.32 & Neary 0 & $999 / 2642$ & $88 / 2159$ & $12 / 3411$ & $1 / 1068$ \\
C2 & 0.474 & 29.22 & Neary 0 & $1055 / 2640$ & $110 / 2067$ & $7 / 3482$ & $3 / 1015$ \\
C3 & 0.506 & 31.45 & Neary 0 & $967 / 2551$ & $81 / 2031$ & $6 / 3696$ & $1 / 1058$ \\
D1 & 0.483 & 27.69 & Neary 0 & $877 / 2351$ & $119 / 1976$ & $10 / 2832$ & $1 / 790$ \\
D2 & 0.494 & 28.09 & Neary 0 & $808 / 2277$ & $111 / 1799$ & $10 / 2975$ & $2 / 736$ \\
D3 & 0.478 & 27.31 & Neary 0 & $844 / 2301$ & $110 / 1830$ & $16 / 2940$ & $0 / 786$ \\
\hline
\end{tabular}

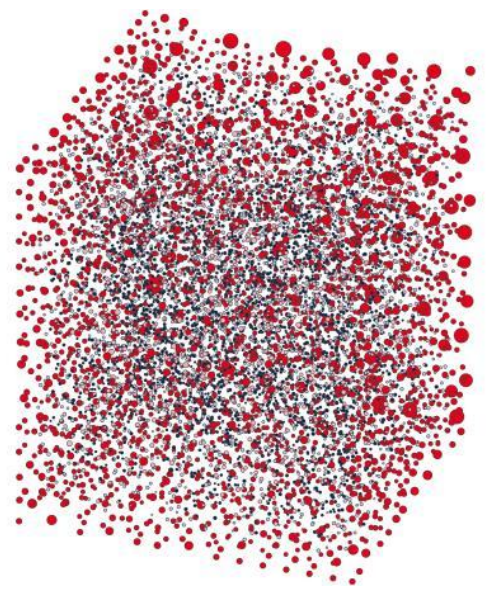

(a) All PBs - A1

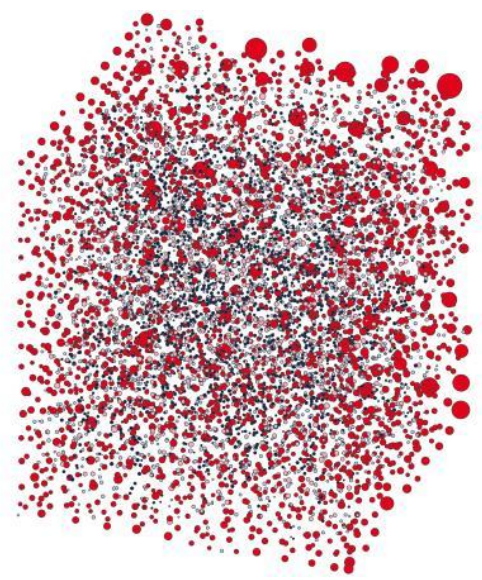

(c)All PBs - D1

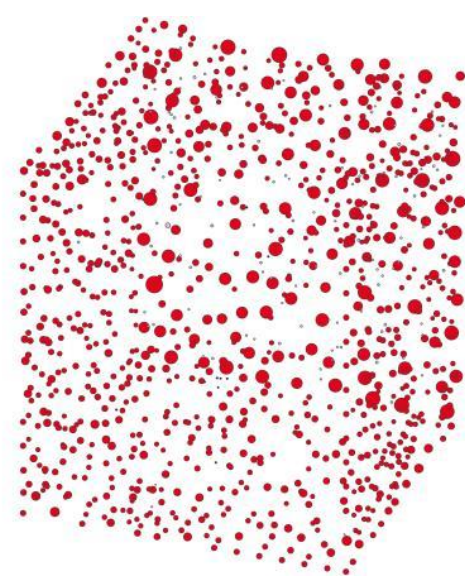

(b) Significant PBs - A1

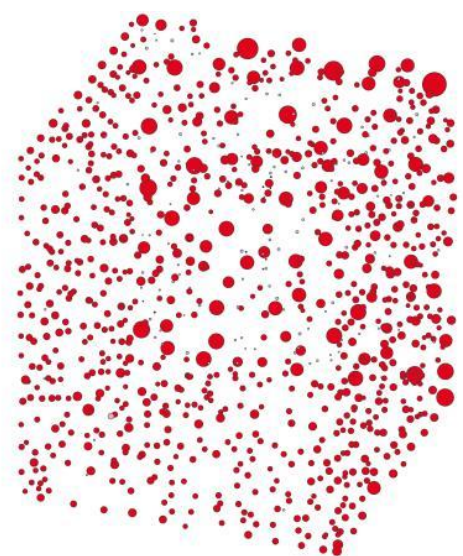

(d) Significant PBs - D1

Fig. 4 Spatial distributions of categories for PB-Internal 


\section{CONCLUSIONS}

In this research, global and local Moran's indexes, which are spatial statistics, are used to analyze the spatial distribution of porous media packed with both single-size and various-size grains. The obtained results of the spatial statistics show that there is a spatial autocorrelation in the pore distribution, both in the porous media packed with single-size spherical grains and uniformly distributed spherical grains. In addition, the numbers of significant $\mathrm{PBs}$ at the 5\% statistically significant level that distributed in $\mathrm{HH}$ category are obviously higher than LH, LL and HL regions, which indicates that large $\mathrm{PBs}$ tend to distribute around large PBs. All of these imply that hydraulic properties of porous media could be affected by the spatial structure of pores.

\section{REFERENCES}

[1] Horton, R., M. D. Ankeny, and R. R. Allmaras, Effects of compaction on soil hydraulic properties, Developments in Agricultural Engineering, Vol. 11, 1994, pp.141-165.

[2] Takeuchi, J., Sumii, W., and Fujihara, M., Modeling of fluid intrusion into porous media with mixed wettabilities using pore-network, International Journal of GEOMATE, Vol. 10, 2016, pp.1971-1977.

[3] Takeuchi, J., Sumii, W., Tsuji, H., and Fujihara, M., Estimation of permeability of porous media with mixed wettabilities using pore-network model, International Journal of GEOMATE, Vol. 11, 2016, pp.2241-2247.

[4] Takeuchi J. and Fujihara M., Spatial Statistical Analysis of Pores in Single-size Grains Packing, Proceedings of JRCSA 2017 Annual Congress, 2017, pp.18-21 (in Japanese).

[5] Moran, P. A., Notes on continuous stochastic phenomena, Biometrika, Vol. 37(1/2), 1950, pp.17-23.

[6] Okabe, A., and Sugihara, K., Network spatial autocorrelation, Spatial analysis along networks: statistical and computational methods. John Wiley and Sons, 2012, pp.137-151.

[7] Pisati, M., sg162: Tools for spatial data analysis, Stata Technical Bulletin, Vol. 60, 2001, pp.21-37.

[8] Rabbani, A., Jamshidi, S., and Salehi, S., An automated simple algorithm for realistic pore network extraction from micro-tomography images. Journal of Petroleum Science and Engineering, Vol. 123, 2014, pp.164-171.

[9] Al-Raoush, R., Willson, C., Extraction of physically realistic pore network properties from three-dimensional synchrotron X-ray microtomography images of unconsolidated porous media systems. J. Hydrol. Vol.300 (1), 2005, pp.44-64.

[10] Gostick, J. T., Versatile and efficient pore network extraction method using marker-based watershed segmentation, Physical Review E, Vol. 96(2), 2017, p.02330.

Copyright (C) Int. J. of GEOMATE All rights reserved, including making copies unless permission is obtained from the copyright proprietors. 